Published in final edited form as:

Nat Biotechnol. 2009 January ; 27(1): 42-44. doi:10.1038/nbt0109-42.

\title{
Crossing the Rubicon
}

\author{
Pedro R Lowenstein \\ Pedro Lowenstein is at the Board of Governors' Gene Therapeutics Research Institute at the \\ Cedars-Sinai Medical Center and the Department of Medicine and Pharmacology at UCLA, 8700 \\ Beverly Blvd., Los Angeles, California 90049, USA. e-mail: lowensteinp@cshs.org
}

\section{Abstract}

A viral vector injected intravenously in mice crosses the blood-brain barrier and transduces widespread regions of the brain and spinal cord.

The gene therapy field has long awaited a viral vector that targets the central nervous system by crossing the blood-brain barrier (BBB). In a watershed paper in this issue, Kaspar and colleagues ${ }^{1}$ show that adeno-associated virus serotype 9 (AAV9) efficiently transduces brain cells located throughout the central nervous system in neonatal mice and, most surprisingly, in adult mice, after intravascular administration. They also provide substantial evidence that AAV9 does so by directly crossing the endothelial BBB. If these results can be reproduced in humans, they may facilitate gene therapy of diseases that affect large areas of the brain, such as motorneuron diseases, Parkinson's disease and Alzheimer's disease.

The BBB constitutes a selective boundary that regulates the entry of ions, large molecules and cells into the brain. It arose early in evolution to maintain brain ionic concentrations between narrow ranges, as early life developed in environments of high salinity ${ }^{2}$. Ions and highmolecular-weight molecules such as antibodies do not readily cross the BBB, whereas nutrients can diffuse across it freely. Certain cells can also cross the BBB, as is seen in infectious or autoimmune brain inflammation. Activated T cells, for example, can cross an intact BBB provided that the specific target antigen for the $\mathrm{T}$ cells is present in the brain. Thus, the barrier is not defined exclusively by size or liposolubility, but serves specific functions ${ }^{3}$.

That $\mathrm{T}$ cells cross the BBB is not always recognized. The best example of this is in adoptive transfer of experimental allergic encephalomyelitis. Following immunization against a brain protein, such as myelin basic protein, transfer of the autoreactive $\mathrm{T}$ cells to a naive host causes experimental allergic encephalomyelitis in the host with a time course almost indistinguishable from that of immunized animals. This demonstrates that activated $\mathrm{T}$ cells can cross the BBB.

Many diseases of the brain could be treated and potentially cured by replacing mutated or missing genes within the affected brain cells. Thus far, experimental and clinical researchers in the field have simply circumvented the BBB by injecting viral vectors directly into the brain and spinal cord. However, direct injections allow transduction of only restricted regions of the brain and require invasive neurosurgery. For gene therapy of diseases that affect large areas of the brain, the preferred approach would be delivery of vectors through systemic administration routes.

Despite considerable efforts to discover viruses or viral gene transfer vectors that cross the $\mathrm{BBB}$, none have yet been identified. In herpes simplex virus (HSV)-1 encephalitis, the virus is thought to enter the brain through the nose, the olfactory bulb and olfactory nerves, a pathway that explains the preference of herpes encephalitis for the limbic system. In the case of HIV encephalitis, the virus enters the brain mainly by hitchhiking across the BBB within infected blood monocytes. Pseudorabies virus can experimentally access the brain from a systemic 
injection site, such as the heart; the pathway used, however, involves retrograde transport from the peripheral organ to the central nervous system through autonomic nerves ${ }^{4}$. And HSV-1 and HSV-2 infections target the oral or genital mucosae from which the virus enters dorsal root ganglion neurons by retrograde transport through peripheral nerve fibers ${ }^{5}$. Thus, viruses that cause encephalitis have evolved access pathways that avoid or fool the BBB. Nevertheless, viruses or bacteria that produce meningitis do reach the meninges from the circulation, as there is no barrier between the blood vessels and the brain coverings.

Gene transfer techniques allow us to modify the genetic makeup of brain cells for therapeutic purposes. Direct injection of viral vectors into the brain has enabled transduction of groups of neurons and astrocytes. Even in cases of diffuse disease, such as Parkinson's disease and Alzheimer's disease, direct delivery of vectors to the striatum, substantia nigra, basal forebrain, subthalamic nucleus and other areas has supported the development of clinical trials using genes encoding various growth factors. Lacking vectors capable of crossing the BBB, researchers have investigated other approaches for achieving widespread transduction, such as convection-enhanced delivery 6 or infection of specific neuronal pathways to distribute therapeutic enzymes globally throughout the brain ${ }^{7}$. Nevertheless, the challenges in targeting large areas of the spinal cord and forebrain remain significant: delivery of vectors through the bloodstream would be the ideal solution.

Past efforts to breach the levees of the brain have used compounds such as bradykinin or nonspecific osmotic opening of the BBB with hyperosmolar mannitol. These techniques are practiced mainly in neuro-oncology to increase the delivery of highly polar chemotherapeutic agents to primary or metastatic brain tumors (such as brain glioblastoma multiforme, primary brain lymphoma or metastatic melanoma to the brain). Although hyperosmotic breaching of the BBB increases entry of viral vectors into the brain ${ }^{8}$, the technique is difficult, cumbersome and risky (as it increases influx of all molecules), and the barrier remains open for only a very short time, precluding massive brain transduction. Thus, these methods are not widely used outside clinical neurooncology.

Kaspar and colleagues ${ }^{1}$ have now demonstrated that delivery of AAV9 through the systemic circulation leads to widespread transduction of the brain in both neonatal and adult mice. These results are of great significance given the longstanding interest in developing vectors that cross the BBB. The authors show that, in neonates, a single injection of AAV9-green fluorescent protein (GFP) into the bloodstream results in broad transduction of the brain, including the spinal cord, the forebrain and the cerebellum; for example, transduction is observed in $\sim 60 \%$ of motor neurons in the spinal cord and of $\sim 65 \%$ of Purkinje cells and $\sim 10-20 \%$ of neurons in the cortex and hippocampus. In adults, $>60 \%$ of spinal-cord astrocytes are transduced, in addition to widespread transduction of astrocytes throughout the forebrain. Such strong transduction throughout large areas of the brain is unprecedented; no previous papers have achieved this in the absence of direct brain injections.

The authors find marked differences in the cell types infected in neonatal and adult mice. In neonates, AAV9 primarily transduces neurons and, secondarily, astrocytes and brain microglia. In adults, it transduces almost exclusively astrocytes and brain blood vessels, although some neuronal transduction is also observed. What accounts for these differences? As AAV9 can clearly cross the endothelial cell layer ${ }^{9}$ that forms the main component of the BBB at both ages, the most likely explanation is the changing nature of the cells that surround the blood vessels and the changing structure and molecular composition of the brain extracellular space between the two ages (Fig. 1).

In neonates, after crossing the endothelial barrier, AAV9 encounters a lax extracellular matrix (possibly with reduced levels of heparan sulfate proteoglycans, which inhibit AAV diffusion 
through the matrix) and can diffuse to enter neuronal axons and dendrites that surround the blood vessels, thereby transducing neurons (Fig. 1). Astrocytes and microglial cells are also present within the extracellular matrix and become transduced as well. In addition, a high ratio of neurons to glia in the neonatal brain must favor neuronal transduction. In contrast, as AAV9 crosses the endothelial barrier in adult mice, it encounters the tightly knit endothelial wrapping of astrocyte end feet (Fig. 1). Thus, it transduces mainly astrocytes and endothelial cells.

Transduction of perivascular pericytes and macrophages is also likely, although this was not examined in detail.

Several results support the authors' claim that AAV9 crosses the BBB. To show that neural cells take up AAV9 rather than GFP, the authors elegantly demonstrate expression of AAV9 mRNA within the brain at both ages. To show that intravascular AAV9 does not reach the brain indirectly through peripheral muscle, they inject the virus directly into the muscle and document poor transduction of the central nervous system. The data presented also suggest that some sort of long-range distribution of AAV9 occurs throughout the neuronal arbors after uptake by axon terminals, given that most transduced cells are those with long axons rather than interneurons. Importantly, transduction was stable over the time period examined of up to 7 weeks.

Thus, Kaspar and colleagues ${ }^{1}$ have demonstrated for the first time diffuse and efficient transduction of the central nervous system from the systemic circulation by a viral vector carrying a marker transgene. Of course, it remains to be determined whether the BBB in humans will be equally permissive to AAV9. However, similarities in the structure and function of the human BBB suggest that this is likely. Moreover, the human brain at birth is more immature than the rodent brain at birth. Thus, it can be extrapolated that AAV9 may provide reasonable brain transduction of human infants, adolescents and even adults. As with all gene therapies, it will be important to address the potential for insertional oncogenesis 10 and adverse immune responses 11 .

If these challenges can be addressed, this work should have important implications for the treatment of various diseases that affect large areas of the central nervous system. These include amyotrophic lateral sclerosis, which progressively affects all motor neurons of the spinal cord; Parkinson's disease, which affects large areas of the basal ganglia, such as the caudate, putamen, substantia nigra and subthalamic nuclei; and Alzheimer's disease, which affects the whole neocortex, the hippocampus and large subcortical regions. The capacity to transduce all main cell types of the central nervous system in neonates suggests that AAV9 could be used to treat young patients with neuronal diseases, such as the spinal muscular atrophies, the neuronal ceroid lipofuscinoses, Huntington's disease and the spinocerebellar degenerations; diseases that affect the microglia, such as the leukodystrophies (e.g., adrenoleukodystrophy, metachromatic leukodystrophy, Krabbe's disease and Canavan's disease); or diseases that affect astrocytes, such as Alexander's disease. In older patients, transduction of astrocytes could provide growth factors to degenerating neurons, as occurs in Parkinson's disease, Alzheimer's disease and amyotrophic lateral sclerosis.

In summary, this study has opened up the exploration of gene therapies that require the transduction of large and diffuse areas of the central nervous system. Having crossed the Rubicon, we now look forward to the next challenges. These include determining whether AAV9 crosses the human BBB, reducing transduction of peripheral organs, targeting AAV9 to subsets of neuronal and glial populations and demonstrating reversion of various brain diseases. Whatever this future research reveals, the work of Kaspar and colleagues ${ }^{1}$ has crossed an important threshold. In the words of Julius Caesar, after traversing the Rubicon into Italy, "Alea iacta est" (the die is cast). 


\section{References}

1. Foust KD, et al. Nat. Biotechnol 2008;27:59-65. [PubMed: 19098898]

2. Lowenstein PR. Trends Immunol 2002;23:23-30. [PubMed: 11801451]

3. Bechmann I, et al. Trends Immunol 28;2007:5-11.

4. E nquist LW. J. Infect. Dis 2002;186:S209-S214. [PubMed: 12424699]

5. Glorioso JC, Fink DJ. Mol. Ther. 2008 October 7; advance online publication

6. Fiandaca MS, et al. Neurotherapeutics 2008;5:123-127. [PubMed: 18164491]

7. Cearley CN, Wolfe JH. J. Neurosci 2007;27:9928-9940. [PubMed: 17855607]

8. Fu H, et al. Mol. Ther 2003;8:911-917. [PubMed: 14664793]

9. Di Pasquale G, Chiorini JA. Mol. Ther 2006;13:506-516. [PubMed: 16368273]

10. Donsante A, et al. Science 2007;317:477. [PubMed: 17656716]

11. Manno CS, et al. Nat. Med 2006;12:342-347. [PubMed: 16474400] 


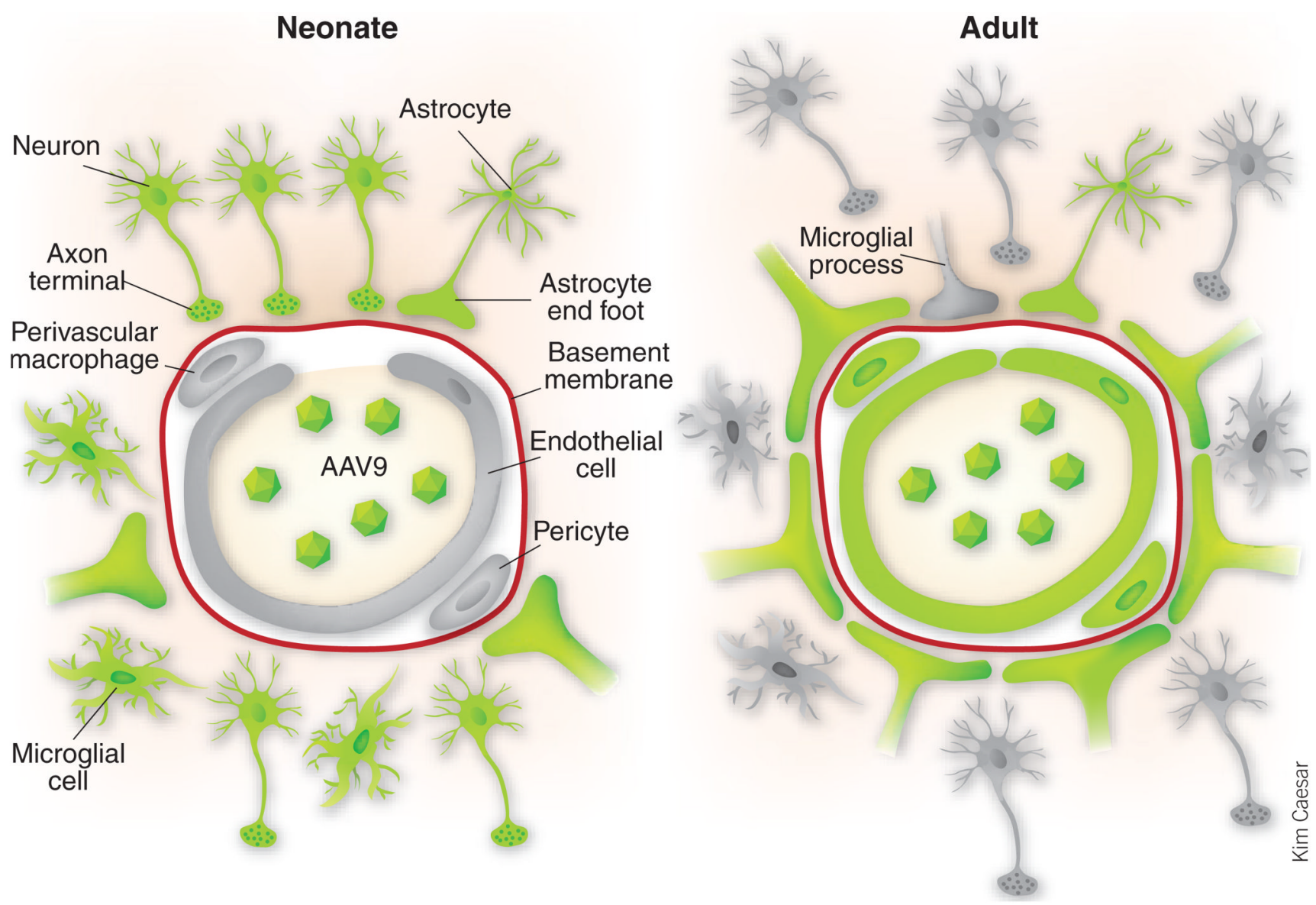

Figure 1.

Developmental changes in the structure of the blood-brain barrier and the brain extracellular matrix likely influence the capacity of AAV9 to transduce different types of brain cells. Transduced cells are shown in green, non-transduced cells in gray. In neonates (left), AAV9 crosses an immature endothelium and diffuses through a lax extracellular matrix to transduce neurons, astrocytes and microglia. In adults (right), the brain endothelium has become a tight barrier surrounded by a cuff of closely packed astrocyte end feet. AAV9 can still cross the brain endothelium but does not seem able to get beyond the glial barrier, thus transducing mainly astrocytes (presumably by infecting end feet) and endothelial cells, and possibly pericytes and perivascular macrophages, but only very few neurons or microglia. 